\title{
"Síndrome pequeño para su tamaño", (small-for-size), como complicación de un trasplante hepático con donante vivo adulto relacionado
}

\author{
Andrés Yarur ${ }^{1}$, Lorena Castro ${ }^{1}$, Roberto Segovia ${ }^{1,2}$, \\ Juan Pablo Roblero', Mario U ribe ${ }^{2}$, Mario Ferrario², \\ Erwin Buckel².
}

\section{Small-for-size syndrome. Report of one case}

\begin{abstract}
Although the use of cadaveric split or living donor liver transplantation is a valid option for liver transplants, they have several complications, being the "small-for-size syndrome" one of the most frequent. This entity is mainly due to the incapacity that the graft has to meet the blood drainage demands. We report a 61 year-old patient with sub-acute liver failure, transplanted with a partial liver graft who developed hyperbilirubinemia, ascites and liver function deterioration. A meso-caval shunt was performed, after which the ascites resolved, serum bilirubin normalized and the synthetic function of the liver improved. After one month, a follow-up CT scan showed the absence of blood flow in the shunt, possible due to the reduction of the hyper-perfusion of the liver. The clinical and biochemical condition of the patient continued improving despite the lack of flow through the shunt (Rev Méd Chile 2009; 137: 918-22).
\end{abstract}

(Key words: Liver failure; Liver transplantation; Portasystemic shunt, surgical)

Recibido el 14 de agosto, 2008. Aceptado el 21 de abril, 2009.

${ }_{1}^{1}$ Departamento de Medicina, Hospital San Borja-Arriarán y ${ }^{2}$ Unidad de Trasplante, Clínica Las Condes, Facultad de Medicina, Universidad de Chile, Santiago de Chile.

$\mathrm{E}$ trasplante de un injerto hepático parcial proveniente de un donante vivo es una práctica utilizada como una potencial solución para las largas listas de espera de trasplante. El empleo de un injerto parcial ha sido realizado tanto con la

$\overline{\text { Correspondencia a: Dr Roberto Segovia. Departamento de }}$ Gastroenterología, Clínica Las Condes. Lo Fontecilla 441, Las Condes. Santiago de Chile. Fono-Fax: 56-2-6108719. E mail: rcasegovia@gmail.com técnica de split liver, como con la donación de una sección hepática de un donante vivo ${ }^{1}$.

Para que el procedimiento sea exitoso se necesita de un injerto que represente al menos $1 \%$ del peso corporal del receptor, por lo cual la procuración de un donante vivo adulto necesita de una hepatectomía derecha, lo que otorga mayor riesgo de morbilidad y mortalidad para el donante, siendo esta última estimada en $0,3 \%$. Es por esto que se necesita encontrar un balance entre el tamaño del injerto utilizado y el riesgo al que se encuentra expuesto el donante ${ }^{2,3}$. 


\section{CASO CĹNICO}

Paciente de 61 años de edad, sexo masculino, minero, con antecedentes de gota en tratamiento con alopurinol y diclofenaco. El día 29 de mayo de 2005 consultó por cuadro de compromiso del estado general e ictericia. En el estudio bioquímico destacaba bilirrubinemia: $20 \mathrm{mg} / \mathrm{dL}$, transaminasa glutámico oxalacítica (GOT): $2.350 \mathrm{U} / \mathrm{L}$, transaminasa glutámico pirúvica (GPT): $1.845 \mathrm{U} / \mathrm{L}$, gamma-glutamil transpeptidosa (GGT): $141 \mathrm{U} / \mathrm{L}$, fosfatasas alcalinas (FA): 187 U/L, INR: 2,8. Tanto los estudios para hepatitis viral y autoinmune, como para enfermedades metabólicas que afectan el hígado (déficit de $\alpha 1$-antitripsina, enfermedad de Wilson y hemocromatosis), resultaron negativos. La tomografía axial computarizada (TAC) abdominal mostró un hígado disminuido de tamaño. La endoscopia digestiva alta evidenció várices esofágicas incipientes. No existía historia de abuso de ingesta de alcohol y tuvo exposición a pesticidas en forma periódica. El paciente negó antecedentes familiares de patología hepática.

El 6 de julio, se trasladó a Santiago con diagnóstico de hepatitis subaguda de etiología no precisada. Clínicamente, se encontraba ictérico, con encefalopatía hepática grado II y ascitis leve. En los nuevos estudios de laboratorio realizados, destacó: inmunoglobulinas: IgG: $1.838 \mathrm{mg} / \mathrm{dL}$ (700-1.600), IgA: $1.193 \mathrm{mg} / \mathrm{dL}$ (70-400), IgM: 118 $\mathrm{mg} / \mathrm{dL}$ (40-230); pruebas hepáticas: bilimubinemina total: 21,9 mg/dL, GOT: 385 U/L, GPT: $202 \mathrm{U} / \mathrm{L}$, FA: $29 \mathrm{U} / \mathrm{L}, \mathrm{GGT}: 320 \mathrm{U} / \mathrm{L}$, albuminemia: $2,6 \mathrm{~g} / \mathrm{dL}$, proteinemia: $3 \mathrm{~g} / \mathrm{dL}$, INR: 2,76. El día 8 de julio se activó para trasplante de urgencia en la lista nacional dado su deterioro progresivo, manteniendo encefalopatía grado II-III, sin embargo, luego de 3 semanas de espera por un órgano cadavérico, se planteó la posibilidad de realizar el trasplante recurriendo a un donante vivo adulto relacionado, que se llevó a cabo el 23 de agosto en Clínica Las Condes, utilizando 2/3 del lóbulo derecho de un sobrino de 36 años, quien fue sometido al protocolo de estudio de donante vivo adulto relacionado y con firma de consentimiento informado, según comité de ética local.

Como inmunosupresión, se utilizó tri-terapia con prednisona, ciclosporina y micofenolato.

El paciente desarrolló una ictericia progresiva y ascitis severa que requirió de paracentesis evacuadora, uso de albúmina y octreotide. A la tercera semana post trasplante los niveles de bilirrubina se mantenían elevados en $27 \mathrm{mg} / \mathrm{dL}$, con un descenso en el tiempo de protrombina a $62 \%$, sin evidenciar alteraciones significativas tanto en el nivel de transaminasas como en las enzimas de colestasis. Cursó 2 episodios de colangitis, en uno de ellos el cultivo biliar resultó positivo para E coli. Fue tratado exitosamente con cefepime y vancomicina. Se realizó una colangiopancreatografía retrógrada y se instaló una endoprótesis por una estenosis biliar en el área de anastomosis. A pesar de lo anterior, los niveles de bilirrubina sérica se mantuvieron elevados. Exámenes de imagenología y una nueva colangiografía descartaron nuevas alteraciones de la vía biliar, aunque una angio-TAC abdominal del 5/9/05 mostró una gran zona hipoperfundida en fase arterial precoz, que en fase tardía se homogenizaba. La arteria hepática se encontraba indemne, sin alteración de las venas hepáticas ni signos de trombosis. El 8/9/05 se efectuó una biopsia hepática que evidenció extensa congestión, hemorragia y necrosis centro lobulillar. Dado la persistencia del cuadro de ascitis a tensión y el deterioro progresivo de la función hepática, el 13 de septiembre, se realizó un shunt meso-cavo con prótesis de $7 \mathrm{M}$ de dacrón en forma términolateral.

El paciente evolucionó con una mejoría significativa en todos sus parámetros. La bilimubinemia disminuyó progresivamente y se evidenció una recuperación del tiempo de protrombina (Tabla 1), con desaparición de la ascitis. Durante los meses siguientes, el paciente fue controlado regularmente, encontrándose parámetros de función hepática normales. La TAC realizada el 19 de octubre de 2005, mostró que el shunt meso-cavo realizado no se encontraba permeable (Figura 1).

El donante fue dado de alta a los 7 días y no ha presentado complicación alguna relacionada a la cirugía luego de 2 años de seguimiento.

\section{DisCUSIÓN}

El uso de un injerto de menor tamaño al necesario o una alteración del sistema de flujo venoso hepático de salida, lo que probablemente ocurrió en este caso, conduce a un cuadro clínico que se 
Tabla 1. Pruebas hepáticas

\begin{tabular}{|lrrrrrrrr|}
\hline & $\mathbf{6 / 7 / 2 0 0 5}$ & $\mathbf{2 6 / 8}$ & $\mathbf{3 0 / 8}$ & $\mathbf{1 2 / 9}$ & $\mathbf{1 7 / 9}$ & $\mathbf{2 6 / 9}$ & $\mathbf{2 / 1 1}$ & $\mathbf{1 / 2 / 2 0 0 6}$ \\
\hline BbT (mg/dL) & 21,9 & 6,3 & 18 & 27 & 14 & 9 & 2,6 & 1,8 \\
ASAT (U/L) & 385 & 205 & 63 & 41 & 30 & 128 & 20 & 32 \\
ALAT (U/L) & 302 & 2.202 & 324 & 29 & 19 & 150 & 36 & 43 \\
FA (U/L) & 29 & 47 & 54 & 243 & 153 & 548 & 232 & 184 \\
GGT (U/L) & 320 & 35 & 97 & 106 & 53 & 280 & 209 & 142 \\
TP (\%) & 31 & 51 & 63 & 48 & 92 & 86 & 90 & 89 \\
INR & 2,76 & 1,72 & 1,44 & 1,80 & 1,08 & 1,13 & 1,12 & 1,14 \\
\hline
\end{tabular}

$\mathrm{BbT}=$ bilirrubina total. ASAT =aspartato aminotransferasa. ALAT =alanina aminotransferasa. FA =fosfatasa alcalina. GGT =gamma glutamil transpeptidasa. TP =tiempo de protrombina

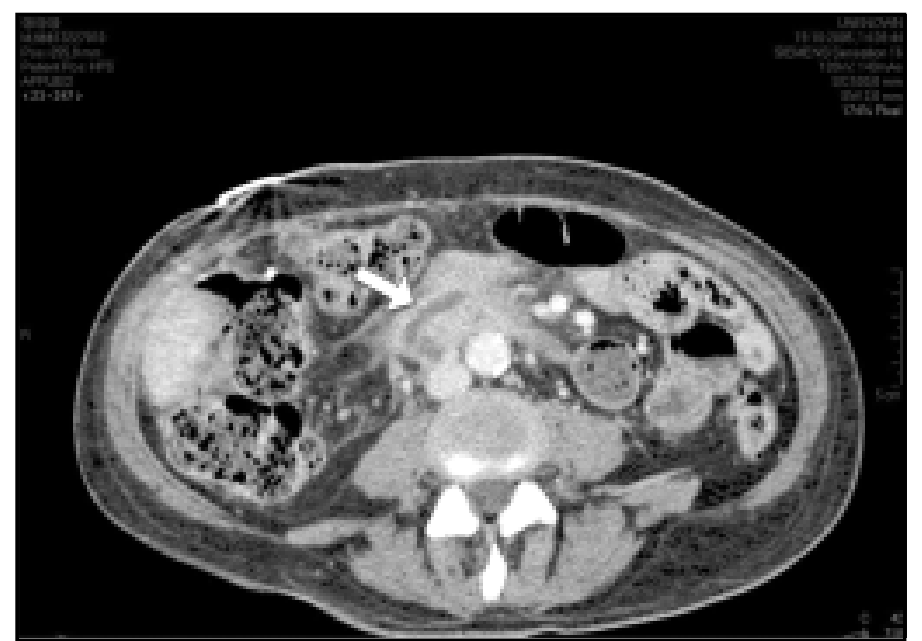

Figura 1. TAC del 19 de octubre, el cual evidencia el shunt meso-cavo sin flujo (flecha).

ha denominado como "síndrome pequeño para su tamaño"4, (small-for-size syndrome), el cual incluye un amplio espectro de entidades clínicas que pueden llevar a la pérdida del injerto y necesidad de re-trasplante. Actualmente no existe un consenso que estructure esta entidad, pero Dahm y cols $^{5}$ proponen subdividirla en 2: Small-for-size non-function y Small-for size dysfunction. La primera es definida como un injerto trasplantado que es incapaz de realizar funciones de síntesis mínimas, con requerimiento de un re-trasplante 0 pérdida del injerto, mientras que a la segunda, como una insuficiencia parcial del hígado trasplantado, con la existencia de 2 de los siguientes parámetros en 3 días consecutivos de la primera semana post trasplante: bilirrubina $>100 \mu \mathrm{mol} / \mathrm{L}$ $(5,84 \mathrm{mg} / \mathrm{dL})$, INR $>2$, encefalopatía grado II o III, donde debería ejercerse alguna intervención terapéutica, descritas a continuación.

Los mecanismos fisiopatológicos responsables de este síndrome no están del todo aclarados, aunque la hipertensión e hiperflujo portal serían las principales causas ${ }^{5,6}$.

Pacientes trasplantados con hígados provenientes de un donante vivo que presenten una relación de peso injerto-paciente menor a 0,8\% exhiben una menor sobrevida, tanto del paciente como del injerto, al compararlos con receptores de injertos de mayor tamaño ${ }^{7}$. También se ha descrito que aproximadamente $20 \%$ de los injertos 
hepáticos parciales evidencian una lesión a la biopsia hepática a los $5 \mathrm{~min}$ de ocurrida la reperfusión del hígado, y este daño es proporcional a la relación peso injerto-paciente ${ }^{8}$.

A pesar de esto, no siempre un injerto proporcionalmente de menor tamaño para el receptor se asocia a la aparición de complicaciones, lo que pone de manifiesto que existen otros factores involucrados en la patogenia de este síndrome. Por ejemplo, se ha descrito alteración en la expresión de genes involucrados en la regulación hemodinámica, en la remodelación celular y en la respuesta al estrés 9 . También se ha planteado que la respuesta inmune en contra del órgano implantado que se encuentra en regeneración pudiera ser mayor que en injertos completos ${ }^{10}$. Los elementos clínicos que podrían influir en el pronóstico y evolución del paciente se muestran en la Tabla $2^{11,12}$.

Las manifestaciones que suelen presentarse son la hiperbilirrubinemia prolongada, ascitis, coagulopatía, daño hepático acelerado, disminución de la función sintética del hígado y colestasis $^{2,5,6}$. Histológicamente, esto se ve representado por un severo balonamiento hepatocitario, colestasis, dilatación centro-lobulillar sinusoidal y atrofia celular ${ }^{13}$.

Existen en evaluación distintas modalidades terapéuticas que intentan disminuir la perfusión y presión portal que estos pacientes presentan. Entre ellas, la experimentación en animales con FK 409 (un liberador de óxido nítrico) y prostaglandina E1 han evidenciado una potencial utilidad, y en un futuro pudieran convertirse en una alternativa para el manejo de estos pacientes ${ }^{14,15}$.
El octreotide, el análogo sintético de la somatostatina, en conjunto o no con esmolol, han sido usados con éxito para reducir la presión y el hiperflujo portal, terapia que no demostró utilidad en este paciente ${ }^{16}$.

En cuanto a las técnicas quirúrgicas, se han ideado varios procedimientos para descomprimir el sistema vascular. La ligadura de la arteria esplénica asociada al banding de la porta y al shunt portosistémico ${ }^{17}$, además del "shunt portocavo término lateral", han demostrado mejorar la sobrevida del paciente y del injerto, previniendo o revirtiendo el síndrome, al reducir la presión portal ${ }^{18}$. En una serie publicada por Gruttadauria y cols ${ }^{19}$, la embolización de la arteria esplénica realizada bajo radioscopia, también se ha mostrado exitosa y reproducible ${ }^{20}$.

En el caso expuesto se observó un cuadro compatible con el síndrome denominado "pequeño para su tamaño", el cual fue revertido con la derivación de un shunt meso-cavo, después de lo cual el paciente evidenció una clara recuperación de sus parámetros bioquímicos y con mejoría clínica de una ascitis de muy difícil control. Es interesante que un mes después del procedimiento, el control radiográfico evidenció una obstrucción completa del shunt (Figura 1). A pesar de lo anterior, las enzimas hepáticas y los niveles de bilirrubina, junto con los factores de síntesis hepática continuaron en franca mejoría. Probablemente esto se debió a que durante el período de tiempo en el cual existió una descompresión de la circulación hepática, el injerto logró fenómenos de regeneración y remodelación vascular, y por lo tanto normalizar su capacidad de drenaje venoso.

Tabla 2. Factores determinantes en la aparición del síndrome "pequeño para su tamaño"

\section{Factores del receptor Factores del injerto/donante}

Patología hepática de base

Enfermedad crónica vs aguda

Edad del receptor

Presencia de ascitis

Presencia de cirrosis hepática

Tamaño esplénico
Edad del donante

Tipo de injerto

Patología latente del injerto

Reconstrucción vascular utilizada

Variación anatómica del injerto

Tiempo de isquemia durante la cirugía 


\section{REFERENCIAS}

1. Middieton P, Duffield M, Lynch S, Padbury R, House T, StANTON P ET AL. Living donor liver transplantationadult donor outcome. Liver Transpl 2006; 12: 24-30.

2. Kiuchi T, Kasahara M, Uryuhara K, Inomata Y, Uemoto S, AsONUMA K ET AL. Impact of graft size mismatching on graft prognosis in liver transplantation from liver donors. Transplantation 1999; 67: 321-7.

3. Malago M, Testa G, Marcos A, Fung JJ, Siegler M, CRONIN DC ET AL. Ethical considerations and rationale of adult-to-adult living donor liver transplantation. Liver Transpl 2001; 7: 921-7.

4. García-ValdeCASAS JC. Trasplante hepático en adulto de donante vivo. Cir Esp 2002; 71: 45-50.

5. Dahm F, Georgiev P, Ciavien PA. Small-for-size syndrome after partial liver transplantation: definition, mechanisms of disease and clinical implications. Am J Transplant 2005; 5: 2605-10.

6. Kiuchi T, Tanaka K, Ito T, Oike F, Ogura Y, Fujimoto Y ET AL. Small-for-size graft in living donor liver transplantation: how far should we go? Liver Transpl 2003; 9: S29-35.

7. Ito $\mathrm{T}$, Kiuchi $\mathrm{T}$, Yamamoto $\mathrm{H}$, Oike $\mathrm{F}$, Ogura $\mathrm{Y}$, Fujimoto Y ET AL. Changes in portal venous pressure in the early phase after living donor liver transplantation: pathogenesis and clinical implications. Transplantation 2003; 75: 1313-7.

8. Keluy DM, Demetris AJ, Fung JJ, Marcos A, Zhu Y, SubBotin V eт AL. Porcine partial liver transplantation: a novel model of the "small-for-size" liver graft. Liver Transpl 2004; 10: 253-63.

9. Man K, Lo CM, Lee TK, L XL, Ng IO, Fan ST. Intragraft gene expression profiles by cDNA microarray in small-for-size liver grafts. Liver Transpl 2003; 9: 425-32.

10. YANG ZF, Ho DW, Chu AC, WANG YQ, FAN ST. Linking inflammation to acute rejection in small-for-size liver allografts: the potential role of early macrophage activation. Am J Transplant 2004; 4: 196-209.

11. Seizner M, Camargo CA, Ciavien PA. Ischemia impairs liver regeneration after major tissue loss in rodents: protective effects of interleukin-6. Hepatology 1999; 30: 469-75.

12. So ejima Y, Mitsuo S, Taketoshi S, Hiroshige S, Ninomiya M, Shiotani S et al. Outcome analysis in adult-toadult living donor liver transplantation using the left lobe. Liver Transpl 2003; 9: 581-6.

13. Demetris AJ, Keliy DM, Eghtesad B, Fontes P, Wawis MARSH J, Tom K. Pathophysiologic observations and histopathologic recognition of the portal hyperperfusion or small-for-size syndrome. Am J Surg Pathol 2006; 30: 986-93.

14. Man K, Lee TK, Lang TB, Lo CM, Fung PC, Tsui SH et al. FK 409 ameliorates small-for-size liver graft injury by attenuation of portal hypertension and down-regulation of Egr-1 pathway. Ann Surg 2004; 240: 159-68.

15. Morioka D, Kubota T, Sekido H, Matsuo K, Satto $\mathrm{S}$, ICHIKAWA Y ET AL Prostaglandin E1 improved the function of transplanted fatty liver in a rat reduced-size-liver transplantation model under conditions of permissible cold preservation. Liver Transpl 2003; 9: 79-86.

16. Busani S, Marconi G, Schiavon L, Rinaldi L, Del Buono M, MasetTi M ET AL. Living donor liver transplantation and management of portal venous pressure. Transpl Proceedings 2006; 38: 1074-5.

17. Troisi $R$, De Hemptinne B. Clinical relevance of adapting portal vein flow in living donor liver transplantation in adult patients. Liver Transpl 2003; 9: S36-41.

18. Troisi R, Ricciardi S, Smeets P, Petrovic M, Van Maeie G, COLE I ET AL. Effects of hemi-portocaval shunts for inflow modulation on the outcome of small-for-size grafts in living donor liver transplantation. Am J Transplant 2005; 5: 1397-404.

19. Gruttadauria S, Mandala L, Miraglia R, Caruso S, MineRvinI MI, Biondo D ET AL. Successful treatment of small-for-size syndrome in adult-to-adult living-related liver transplantation: single center series. Clin Transplant 2007; 21: 761-6.

20. TaKada $Y$, Ueda M, Ishikana $Y$, Fujimoto $Y$, Mirauchi $H$, OgURA Y ET AL. End-to-side portocaval shunting for a small-for-size graft in living donor liver transplantation. Liver Transpl 2004; 10: 807-10. 